\title{
Effects of Bacillus subtilis on performance, immune system and gut in Salmonella- challenged broilers
}

\author{
A. Sikandar ${ }^{1 \#}$, H. Zaneb ${ }^{2}$, A. Nasir ${ }^{3}$, M. Adil ${ }^{1}$, H. M. Ali ${ }^{4}$, N. Muhammad ${ }^{1}$, T. $\operatorname{Rehman}^{4}$, \\ A. Rehman ${ }^{5}$ \& H. F. Rehman ${ }^{2}$ \\ ${ }^{1}$ Department of Basic Sciences, College of Veterinary and Animal Sciences (CVAS), Jhang, sub-campus, University of \\ Veterinary and Animal Sciences (UVAS), Lahore, 35200, Pakistan \\ ${ }^{2}$ Department of Anatomy and Histology, UVAS, 54000, Lahore, Pakistan \\ ${ }^{3}$ Department of Clinical Sciences, CVAS, Jhang, sub-campus, UVAS, Lahore, 35200, Pakistan \\ ${ }^{4}$ University College of Veterinary and Animal Sciences, The Islamia University of Bahawalpur - 63100, Pakistan \\ ${ }^{5}$ Department of Pathobiology, CVAS, Jhang, sub-campus, UVAS, Lahore, 35200, Pakistan
}

(Submitted 25 January 2020; Accepted 18 September 2020; Published 9 October 2020)
Copyright resides with the authors in terms of the Creative Commons Attribution 4.0 South African Licence.
See: http://creativecommons.org/licenses/by/4.0/za
Condition of use: The user may copy, distribute, transmit and adapt the work, but must recognise the authors and the South African Journal of Animal Science.

\begin{abstract}
The goal of this study was to compare the effects of Bacillus subtilis and the antibiotic enrofloxacin on growth performance, immune response, intestinal histomorphometry and cecal Salmonella numbers in broilers challenged with $S$. gallinarum. Salmonella-free chicks $(n=240)$ were allocated to four groups, comprising six replicates $(n=10)$ each. Group NN was not infected and received no treatment. Group SN was infected with Salmonella and received no treatment. Group SE was infected with Salmonella and treated with the antibiotic enrofloxacin. Group SP was infected with Salmonella and treated orally with the probiotic $B$. subtilis. Initially the groups that were infected with Salmonella had reduced growth performance compared with NN. By the fourth week, SE and SP had recovered and weighed as much as or more than NN. The SP group had greater cellular immunity and relative weights of the bursa and thymus than the other groups. SN had the lowest villus height in all the segments of the small intestine and a larger Salmonella population in the ceca. Probiotic supplementation reduced the pathological outcomes more than enrofloxacin. Prophylactic use of $B$. subtilis-type probiotics had similar effects to the use of antibiotics, alleviated the stress related to infection of $S$. gallinarum, and improved the growth performance, immune function, and gut mucosal development in broilers.
\end{abstract}

Keywords: growth promoters, immunity, probiotics, poultry villi length

\#Corresponding author: e-mail: arbab.sikandar@uvas.edu.pk

\section{Introduction}

Salmonellosis is one of the leading causes of lost productivity in poultry (Saleem et al., 2016) and a major source of bacterial food poisoning in humans. Antibiotics, including enrofloxacin, have shown promising efficacy in vitro against the bacterium, and enhance the growth performance of poultry (Xie et al., 2017). Nevertheless, the European Union (EU) withdrew the use of antibiotics in food animals to circumvent the emergence of resistant pathogenic organisms and their transmission to humans through the consumption of contaminated food (Dersjant-Li et al., 2014). This prohibition led to a decline in animal performance from bacterial stress and reduced growth. Consequently, probiotics (Yang et al., 2018), organic acids (Arbab et al., 2017), enzymes (Dersjant-Li et al., 2016), and medicinal plants (Yasmin et al., 2019) are regarded as replacements for antibiotics to enhance the production of poultry.

Probiotics are living cultures of microorganisms that are intended to have health benefits when consumed. B. subtilis is a probiotic that is an effective growth promoter and is capable of enhancing production and improving the innate immune response of broiler chickens (Lee et al., 2015; Tarradas et al., 2020). B. subtilis produces antimicrobial substances and is assumed to facilitate gut health (Sikandar et al., 2017; Abudabos et al., 2019). It favours the growth of lactic acid, producing bacteria and other beneficial microorganisms in the gut that act to competitively exclude pathogens (Yang et al., 2018). However, there is a shortage of information about the protective effect of $B$. subtilis (QST-713) on the gut histology of and other 
health benefits for broilers infected with Salmonella pathogens. Therefore, the goal of the current research was to evaluate the potential effects of $B$. subtilis on growth, gut morphology and the immune system response in broiler chickens challenged with S. gallinarum.

\section{Materials and Methods}

Procedures and protocols used in this study were approved by the Ethical Review Committee for Use of Laboratory Animals of the University of Veterinary and Animal Sciences, Lahore (Notification No. DR/257). Pathogen-free day-old male Hubbard broiler chicks (M77) from Big Bird Group (Lahore, Pakistan) of approximately equal weight $(n=240)$ were randomly divided into four groups, each comprising six replicates of ten chicks. Group NN was not infected and received no treatment. Group SN was infected with Salmonella and received no treatment. Group SE was infected with Salmonella and treated with the antibiotic enrofloxacin. Group SP was infected with Salmonella and treated orally with the probiotic $B$. subtilis. The birds that were assigned to the infected groups were given the Salmonella orally. The groups were housed separately in environmentally controlled closed poultry units that had been disinfected before the arrival of the chicks. The basal diets for the initial ( 1 to 21 days) and finishing (22 to 35 days) phases were as described by Sikandar et al. (2017). These diets were autoclaved at $120^{\circ} \mathrm{C}$ for 15 minutes when each batch of feed was prepared. Minerals, vitamins, amino acids, probiotics, and organic acid were added to each batch, which was then mixed for 10 minutes. Husbandry practices and the biosecurity measures were optimized during the experiment. Feed, in mash form, and water were provided ad libitum. All the birds were monitored for general health conditions and behavioral alterations thrice a day throughout the trial.

Before the start of the experiment, three birds were randomly selected and killed to collect their cecal tonsils. The organs were removed aseptically and put in test tubes containing $10 \mathrm{ml}$ tetra-thionate broth and kept overnight in the incubator at $37^{\circ} \mathrm{C}$. The samples were then plated on brilliant green agar (CM0263, Thermo Fisher Scientific Inc., Waltham, Massachusetts, USA) and again incubated overnight at $37^{\circ} \mathrm{C}$. These plates were then observed for the presence or absence of Salmonella infection in the birds.

A local isolate of $S$. gallinarum (isolated from poultry gut) and maintained at the University Diagnostic Laboratory (ISO certified) was used in the current study. The organism was retrieved on MacConkey broth from a freeze-dried culture of Salmonella seed and then transferred to Salmonella Shigella agar (SS agar) for purification. The culture was then incubated overnight at $37^{\circ} \mathrm{C}$ in Tryptone Soya Broth (Thermo Fisher Scientific Inc., Waltham, Massachusetts, USA) prior to inoculation in the chicks. The infection was introduced in chicks at day 3 via oral gavage at the rate of $0.2 \mathrm{ml}$ suspension of $S$. gallinarum per bird, which contained approximately $10^{7} \mathrm{cfu} / \mathrm{chick}$. The NN group was administered the same amount of sterile buffered peptone water (Sigma-Aldrich Inc., St. Louis, Missouri, USA).

The birds in SP were treated continuously with B. subtilis QST-713 (Agraquest ${ }^{\circledR}$ Bayer, Davis, California, USA), which was provided in the feed at the rate of $0.1 \mathrm{~g} / \mathrm{kg}$ of feed (approximately $2.0 \times 10^{10}$ $\mathrm{cfu} / \mathrm{g}$ ). Birds in SE followed Randall et al. (2006), except that $50 \mathrm{ppm}$ enrofloxacin (10\% solution (Symans Pharmaceuticals Pvt. Ltd., Lahore, Pakistan) was added to the drinking water on days $5-12$ at a ratio of $1 \mathrm{ml}$ drug/ $2 \mathrm{~L}$ water. If the medicated water was completely consumed in a day, then fresh water was made available. All chicks were vaccinated for Newcastle Disease (Nobilis ${ }^{\circledR}$ ND LaSota, Intervet International B.V. Boxmeer, Holland) with the vaccination schedule following industry-standard practice.

The average live bodyweight of each replicate was recorded at the beginning of each experimental week in the early morning before the birds were fed. Weekly weight gain was calculated, and feed intake was recorded weekly to estimate the feed conversion ratio (FCR) (as g feed: $g$ gain) for each replication (Abo Ghanima et al., 2020). The experimental unit was observed strictly for deaths of the birds. When a mortality occurred, the bird was weighed and removed promptly. Mortality rate was calculated weekly as a cumulative percentage. All data were adjusted to account for the mortality.

Cell-mediated immunity was measured according to Sikandar et al. (2017). On day 17, the basophilic hypersensitivity test was administered to two apparently healthy birds from each replicate by intradermal injection between the third and fourth digits of the right foot with $100 \mu \mathrm{g} / 100 \mu \mathrm{l} / \mathrm{bird}$ with phytohaemagglutinin-P (PHA-P) (Sigma-Aldrich, St. Louis, Missouri, USA). For comparison, $100 \mu$ l sterile phosphate-buffered saline was injected in the left foot and served as a control. The swelling at the site of injection was measured at 24 hours, 48 hours and 72 hours post injection with a pressure sensitive micrometer. Finally, the cell-mediated immune response (foot web index) to PHA-P was analysed by subtracting the thickness of the left foot from that of the right foot.

The organism was quantified in the cecal contents according to Park et al. (2014) with slight modifications. Briefly, six birds from each treatment were slaughtered, and the ceca were isolated aseptically. One gram digesta was taken from each cecum and mixed in sterile normal saline $(\mathrm{NaCl} 0.9 \%$ $\mathrm{w} / \mathrm{v})$. About $2 \mathrm{ml}$ sample material of this cecal solution was added to peptone water and incubated overnight at $37 \stackrel{\circ}{\circ}$. Subsequently, a tenfold serial dilution $(1: 9, \mathrm{w} / \mathrm{v})$ was prepared and $50 \mu \mathrm{l}$ of peptone water 
(collected at the seventh dilution) was poured on SS agar. After 24 hours incubation, colony forming units (cfu) were counted in triplicate and the data were expressed as $\log ^{10} \mathrm{cfu} / \mathrm{gm}$.

One bird was selected randomly from each replicate, weighed, and killed on days 21 and 35 . Visceral organs, including the small intestine, liver, thymus, spleen and bursa of Fabricius, were removed and weighed. Samples of intestinal tissue $(0.5-1.0 \mathrm{~cm})$ were subsequently collected and preserved in $10 \%$ neutral buffered formalin for further histo-pathological analysis. The tissues excised from the duodenum (10 $\mathrm{cm}$ distal to the duodeno-gizzard junction), jejunum (5 $\mathrm{cm}$ proximal to Meckel's diverticulum), and ileum (5 $\mathrm{cm}$ anterior to the ileo-cecal junction) were processed with the paraffin sectioning technique. Histological sections of about $5 \mu \mathrm{m}$ thickness were prepared with a semi-automated rotary microtome (AMOS Scientific AEM-450, Austria) and stained with Haematoxylin and Eosin (Sikandar et al., 2012). Three independent sections of each intestinal tissue were selected (about $50 \mu \mathrm{m}$ apart) and five well-oriented villi with intact lamina propria were selected for morphometric analysis. The slides were examined under a light microscope (Olympus CX31, Olympus Corp. Centre Valley, Pennsylvania, USA) at 4x magnification and analysed with a digitalized live image analysis program (Olympus DP20, Olympus Corp. Centre Valley, Pennsylvania, USA) and villus height was determined.

The normal distribution of the data was confirmed with the Kolmogorov-Smirnov test. The data were analysed using one-way ANOVA (SPSS Inc., version 20, Chicago, IL, USA). Each replicate was considered an experimental unit. Differences among means were assessed using Duncan's multiple range test. Effects were considered significant at $P \leq 0.05$. Results are presented as mean $\pm \mathrm{SE}$.

\section{Results and Discussion}

Salmonellosis is a worldwide problem of the poultry sector, particularly in developing countries (Saleem et al., 2016). Probiotics play an important role In combatting its invasion, and not only improve growth performance, but protect the mucosal epithelium from bacterial invasion, and hence impede its growth in tissues. The probiotic $B$. subtilis, which is added as a feed supplement, is gaining popularity in poultry production across the world by boosting the immune response (Dersjant-Li et al., 2014). The positive effect of B. subtilis in non-infected healthy chickens was reported elsewhere (Sikandar et al., 2017). However, limited studies have emphasized the prophylactic effects of $B$. subtilis QST-713 in birds that are challenged with Salmonella gallinarum. Therefore, the present project was designed to observe the influence of $B$. subtilis on broiler health and performance in Salmonella-challenged birds.

Birds of the infected groups had higher $(P<0.05)$ FCR during the first and second weeks compared with NN, whereas in the third week the FCR was higher $(P<0.05)$ for SN compared with other groups (Table 1). During the starter and grower phases, the FCR was statistically lower $(P<0.05)$ in non-infected NN than other groups, whereas during the finisher phase, higher $(P<0.05)$ growth performance was observed in SE, $\mathrm{SP}$ and NN compared with SN group (Table 1).

Table 1 Effects of Bacillus subtilis and Enrofloxacin on growth performance of broilers

\begin{tabular}{|c|c|c|c|c|c|c|}
\hline \multirow{2}{*}{$\begin{array}{l}\text { Age, } \\
\text { week }\end{array}$} & \multirow{2}{*}{ Measurement } & \multicolumn{5}{|c|}{ Treatments } \\
\hline & & NN & SN & SE & $\mathrm{SP}$ & $P$-value \\
\hline \multirow{2}{*}{1} & Gain & $94.33 \pm 2.60^{\mathrm{a}}$ & $68.00 \pm 2.65^{b}$ & $72.00 \pm 2.52^{b}$ & $65.67 \pm 1.20^{\mathrm{b}}$ & 0.000 \\
\hline & FCR & $1.36 \pm 0.03^{\mathrm{b}}$ & $1.84 \pm 0.08^{\mathrm{a}}$ & $1.66 \pm 0.07^{\mathrm{a}}$ & $1.78 \pm 0.07^{\mathrm{a}}$ & 0.002 \\
\hline \multirow{2}{*}{2} & Gain & $241.00 \pm 2.00^{a}$ & $51.00 \pm 0.58^{\mathrm{c}}$ & $96.33 \pm 5.78^{b}$ & $109.33 \pm 2.40^{\mathrm{b}}$ & 0.000 \\
\hline & FCR & $1.29 \pm 0.02^{d}$ & $4.76 \pm 0.04^{\mathrm{a}}$ & $3.07 \pm 0.20^{b}$ & $2.50 \pm 0.06^{c}$ & 0.000 \\
\hline \multirow{2}{*}{3} & Gain & $272.00 \pm 6.66^{a}$ & $71.00 \pm 7.55^{\mathrm{d}}$ & $220.33 \pm 4.91^{c}$ & $242.67 \pm 5.33^{\mathrm{b}}$ & 0.000 \\
\hline & FCR & $2.07 \pm 0.07^{b}$ & $5.05 \pm 0.34^{\mathrm{a}}$ & $2.25 \pm 0.03^{b}$ & $2.07 \pm 0.04^{b}$ & 0.000 \\
\hline \multirow{2}{*}{4} & Gain & $314.67 \pm 7.51^{b}$ & $156.00 \pm 17.09^{c}$ & $494.67 \pm 20.85^{a}$ & $520.67 \pm 16.46^{\mathrm{a}}$ & 0.000 \\
\hline & FCR & $2.45 \pm 0.06^{b}$ & $3.93 \pm 0.41^{\mathrm{a}}$ & $1.51 \pm 0.09^{c}$ & $1.40 \pm 0.03^{\mathrm{c}}$ & 0.000 \\
\hline \multirow[b]{2}{*}{5} & Gain & $461.66 \pm 60.92^{a}$ & $219.67 \pm 20.41^{b}$ & $517.67 \pm 26.44^{\mathrm{a}}$ & $480.33 \pm 21.76^{a}$ & 0.000 \\
\hline & FCR & $2.64 \pm 0.35^{b}$ & $4.28 \pm 0.38^{\mathrm{a}}$ & $2.14 \pm 0.13^{\mathrm{b}}$ & $2.26 \pm 0.08^{\mathrm{b}}$ & 0.002 \\
\hline
\end{tabular}

${ }^{a, b, c}$ Within a row, means with a common superscript were not different at $P=0.05$

NN: not-infected, not medicated; SN: S. gallinarum infected, not medicated; SE: S. gallinarum infected, medicated with 50 ppm enrofloxacin; SP: S. gallinarum infected, mediated with $0.1 \mathrm{~g} / \mathrm{kg}\left(2.0 \times 10^{10} \mathrm{cfu} / \mathrm{gm}\right)$ B. subtilis. 
Supplementation of $B$. subtilis ameliorated the growth performance of the infected birds and the birds of the supplemented groups were partially capable of adjusting the stress caused by the infection. Salmonella infection undesirably exaggerated the growth performance of the infected birds related to noninfected NN group. This reduction in the growth could be attributed to retarded gut function and feed malabsorption due to luminal viscous conditions caused by inflammation and sloughing of the intestinal mucosa (Sikandar et al., 2013). The supplemented groups showed lower $(P<0.05)$ FCR from the third week onwards, which indicated that the products used in those groups exerted a protective effect against Salmonella and thus reflected a better feed efficiency. Lower FCR and better growth in the supplemented groups could be correlated with villus height in the small intestine (Dersjant-Li et al., 2016), because increased size of villi provides a bigger area for the absorption of available nutrients, whereas the challenged group has the lowest height of intestinal villi. A lower feed intake in SN infected birds might be the reason for decreased weight gain. Consequently, this reduced feed intake, in conjunction with poor absorption of feed through dwarfed villi, contributed to compromised immunity in infected chickens.

The thickness of the right foot was significantly higher $(P<0.05)$ in SP at all 24 hour, 48 hour and 72 hour intervals post injection compared with other groups after injecting phyto-haemagglutinin-P (Figure 1).

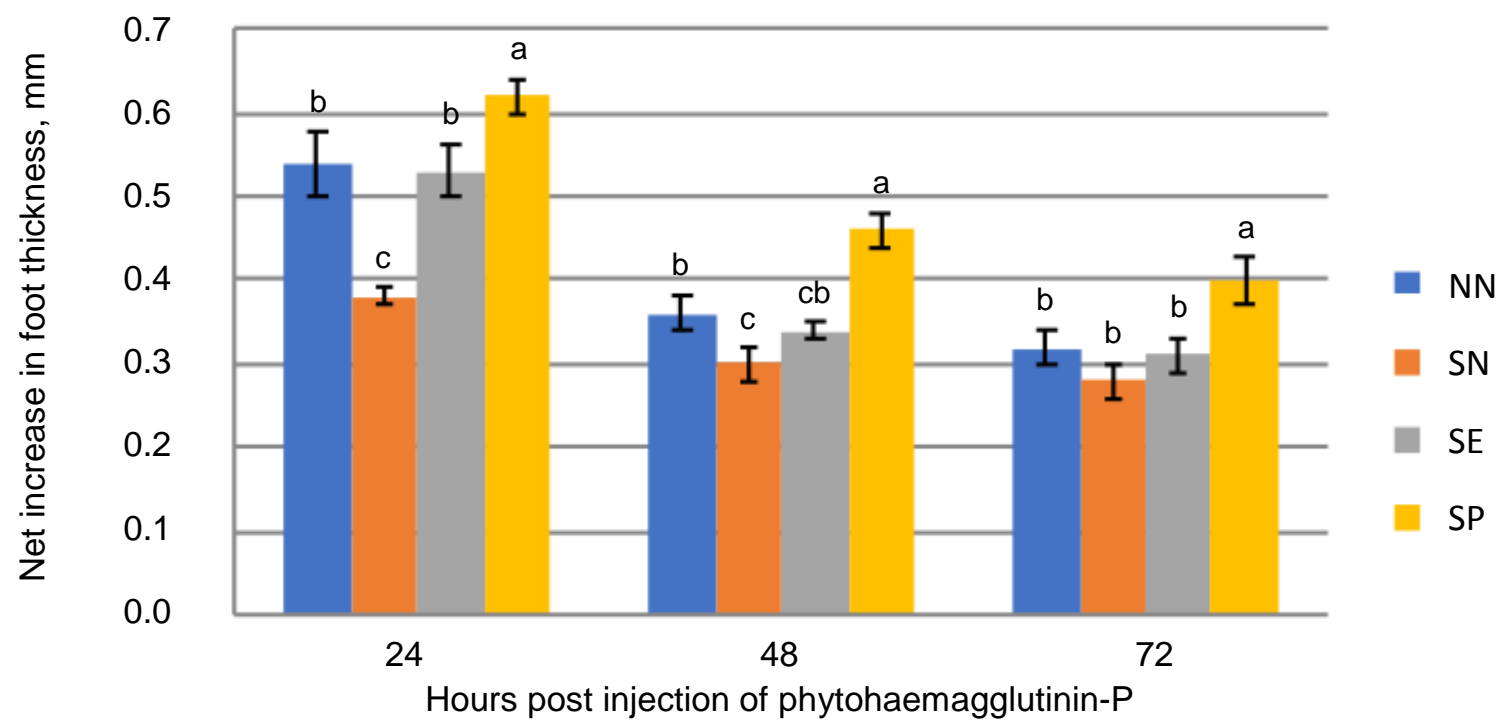

Figure 1 Lymphoproliferative immune response post injection of phytohaemagglutinin- $\mathrm{P}$ in Salmonellainfected and control broiler chicks

Within a time point, bars headed by a common letter were not different at $P=0.05$

NN: non-infected, non-medicated; SN: S. gallinarum challenged, non-medicated; SE: S. gallinarum challenged +50 ppm enrofloxacin; SP: S. gallinarum challenged $+0.1 \mathrm{~g} / \mathrm{kg}\left(2.0 \times 10^{10} \mathrm{cfu} / \mathrm{gm}\right)$ B. subtilis.

The thicknesses of the foot and organ-body weight ratio in infected animals indicated lower cellmediated immunity. But the high cell-mediated immune response post PHA-P injection indicated that $B$. subtilis played a role in the stimulation of an immune response to an acute infection. It is well recognized that B. subtilis has a role in the modulation of normal microflora (Zhang et al., 2017), and regulation of systemic immuno-modulatory functions in healthy chickens (Lee et al., 2015; Tarradas et al., 2020), and in those infected with coccidiosis (Dersjant-Li et al., 2016).

On day 21, the relative weight of thymus was decreased $(P<0.05)$ in $\mathrm{SN}$, whereas that of bursa increased $(P<0.05)$ in SP compared with the NN and SN groups (Figure 2). Compared with other groups, the relative weight of thymus was higher $(P<0.05)$ in SP on day 35 (Figure 3 ). 


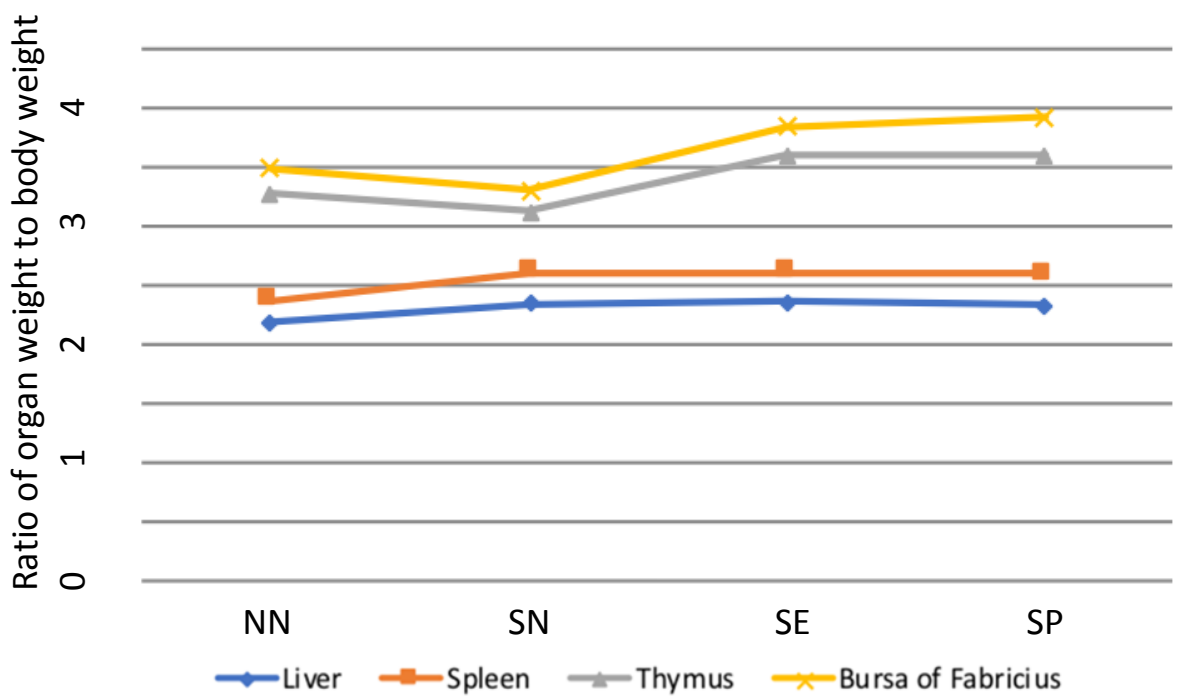

Figure 2 Ratio of organ weight to bodyweight for lymphoid organs harvested from broiler chickens on day 21

NN: non-infected, non-medicated; SN: S. gallinarum challenged, non-medicated; SE: S. gallinarum challenged +50 ppm enrofloxacin; SP: S. gallinarum challenged $+0.1 \mathrm{~g} / \mathrm{kg}\left(2.0 \times 10^{10} \mathrm{cfu} / \mathrm{gm}\right)$ B. subtilis

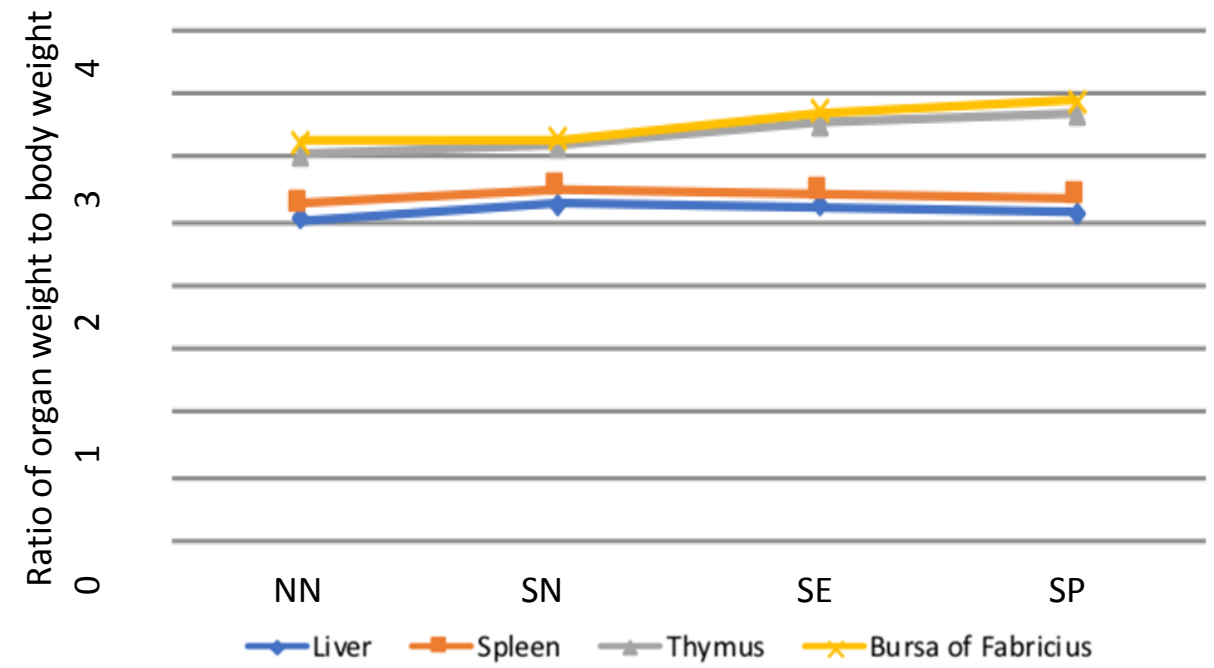

Figure 3 Ratio of organ weight to bodyweight for lymphoid organs harvested from broiler chickens on day 35

NN: non-infected, non-medicated; SN: S. gallinarum challenged, non-medicated; SE: S. gallinarum challenged + 50 ppm enrofloxacin; SP: S. gallinarum challenged $+0.1 \mathrm{~g} / \mathrm{kg}\left(2.0 \times 10^{10} \mathrm{cfu} / \mathrm{gm}\right) B$. subtilis

The relative weights of thymus and bursa decreased in the $\mathrm{SN}$ group but increased with supplementation of $B$. subtilis. This enhanced weight might be owing to a compensatory mechanism by which an increased number of antibodies were produced to combat the disease condition (Shabani et al., 2019). Differences were not detected among groups in the relative weights of liver and spleen, as observed elsewhere (Saleem et al., 2016). The spleen and liver of the SN group were moderately swollen with oedema, and surface capsules were fragile, cracked on the application of gentle pressure, and had yellowish streaks on the surface.

The microarchitecture of the gut mucosa, including villus height, represents a well-known histological parameter to assess the digestive and absorptive efficiency in the intestine (Stefanello et al., 2020). These structures are exposed primarily to feed and its antigens cause lesions and sloughing of the epithelial cells and thus 
affect their secretary and absorptive functions (Sikandar et al., 2012). In the duodenum the villus height decreased $(P<0.05)$ in $\mathrm{SN}$ compared with all other groups at day 21 (Figure 4).

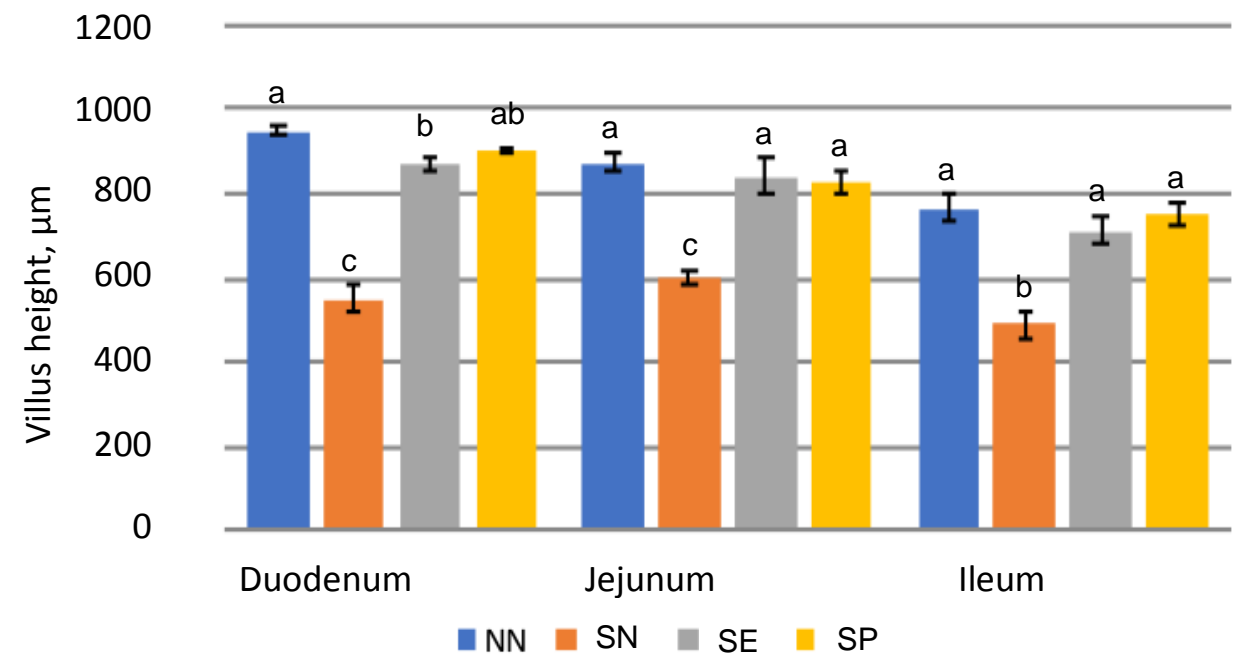

Figure 4 Villus heights for segments of small intestine in Salmonella-infected broiler chicks and control chicks on day 21

a,b,c Within a segment, bars headed by a common letter were not different at $P=0.05$

NN: non-infected, non-medicated; SN: S. gallinarum challenged, non-medicated; SE: S. gallinarum challenged +50 ppm enrofloxacin; SP: S. gallinarum challenged $+0.1 \mathrm{~g} / \mathrm{kg}\left(2.0 \times 10^{10} \mathrm{cfu} / \mathrm{gm}\right)$ B. subtilis.

At day 35, the villus height of duodenal mucosa was greater $(P<0.05)$ in SP compared with NN and SN groups (Figure 5 ) whereas the villus length was lower $(P<0.05)$ in $\mathrm{SN}$ compared with all other groups for the jejunum and ileum.

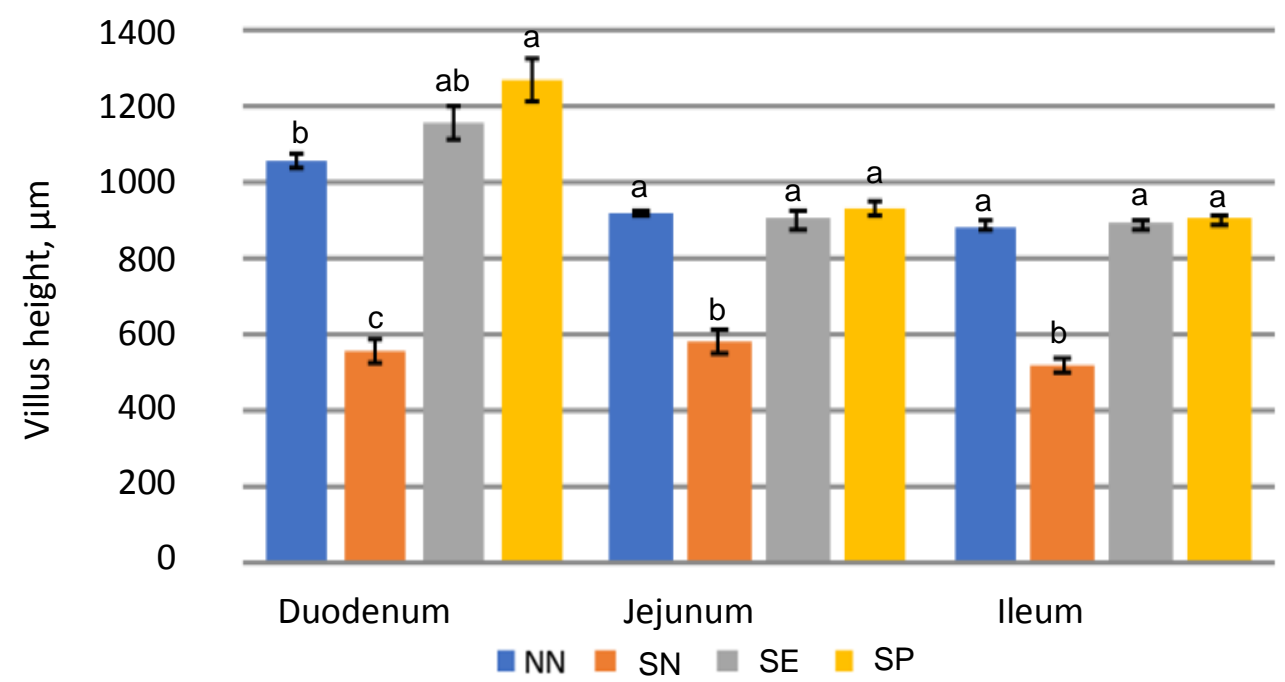

Figure 5 Villus heights for segments of small intestine in Salmonella-infected broiler chicks and control chicks on day 35

${ }^{\text {a,b,c }}$ Within a segment, bars headed by a common letter were not different at $P=0.05$

NN: non-infected, non-medicated; SN: S. gallinarum challenged, non-medicated; SE: S. gallinarum challenged + 50ppm enrofloxacin; SP: S. gallinarum challenged $+0.1 \mathrm{~g} / \mathrm{kg}\left(2.0 \times 10^{10} \mathrm{cfu} / \mathrm{gm}\right)$ B. subtilis

In the current study, the increases in villus height of the intestinal segments in the groups supplemented with $B$. subtilis indicated an underlying protective mechanism comparable with that in the SE group. Moreover, the improved morphometric indices of the gut segments in SP might be attributable to an 
increase in the number of microbiomes in sympathetic association with the mucosa (Awad et al., 2016) and enhanced vascular supply in reaction to Salmonella in the intestinal lumen. An increase in the surface area of the villi (not reported) in the jejunum and ileum in all supplemented groups compared with SN indicated the enhanced villus function that resulted in better performance by the supplemented groups. Deeper crypts (not reported) in the SN group might indicate rapid tissue turnover following mucosal renewal to maintain the absorptive surface area (Yang \& Liao, 2019). The tissue that was most affected was the lining mucosa of the small intestine in the affected SN group, in which the epithelium lining the villi was sloughed, and the villi were reduced in size, and appeared to be denuded and ulcerated. This caused a reduction of villus length and the surface area available for nutrient uptake that might ultimately be linked with the lower growth rate in the SN group.

Because increased $(P<0.05)$ numbers of Salmonella were present in the $S N$ group, this confirmed the success of the challenge. On days 21 and 35 the protective effect of SP was similar to that of the SE group in controlling the cecal Salmonella population and load (Figure 6).

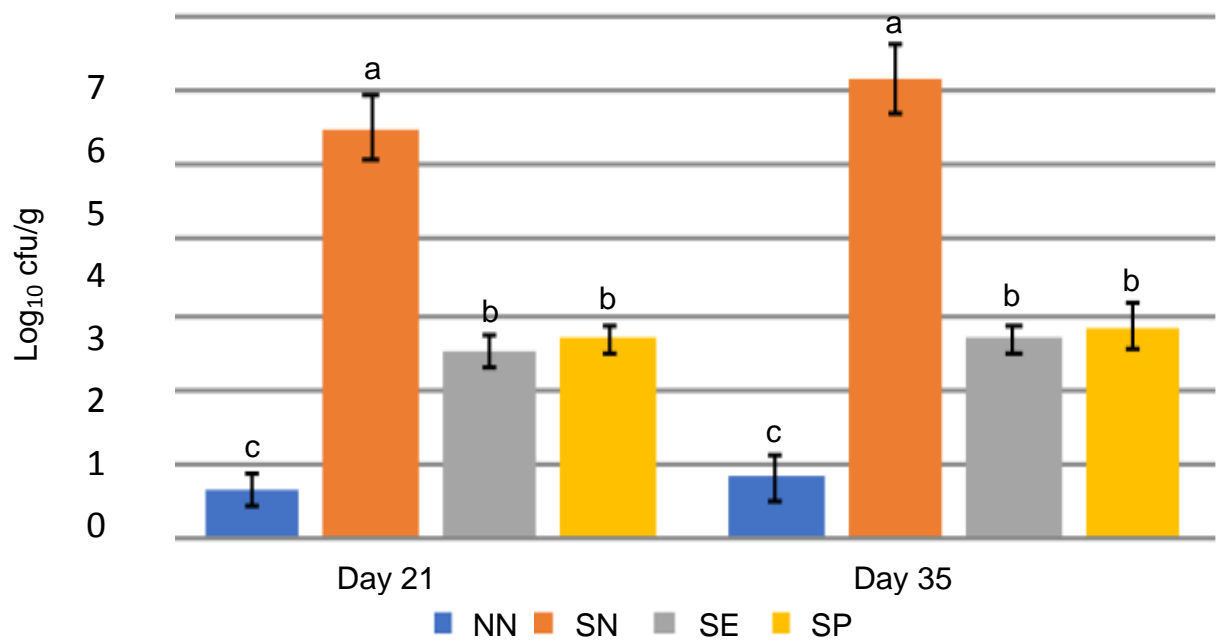

Figure 6 Quantification of intestinal Salmonella numbers in broiler chicks treated with antibiotic or probiotic and control chicks

a,b,c Within a day, bars headed by a common letter were not different at $P=0.05$

NN: non-infected, non-medicated; SN: S. gallinarum challenged, non-medicated; SE: S. gallinarum challenged +50 ppm enrofloxacin; SP: S. gallinarum challenged $+0.1 \mathrm{~g} / \mathrm{kg}\left(2.0 \times 10^{10} \mathrm{cfu} / \mathrm{gm}\right)$ B. subtilis.

The ceca are the preferred site of Salmonella and analysed for the effects of treatments against the bacterium (Nair et al., 2019). Salmonella was isolated from all the replicates in this study, and thus the supplemented ingredients were not able to prevent its colonization in the ceca. However, significant reductions in the size of the Salmonella populations were observed in the SE and SP groups with greater $(P$ $<0.05)$ colonization being found in the SN group. These results are in line with the findings of Saleem et al. (2016) and Arbab et al. (2017), in which higher colonization of Salmonella was observed in the ceca after an orally administered challenge. Spores of $B$. subtilis are suitable candidates for the competitive exclusion of the pathogens (de Oliveira et al., 2014). Moreover, it has been reported that $B$. subtilis causes the expression of Interleukin 22 and the inhibitor of nuclear factor kappa B in the mucosa for defence against harmful microorganisms (Yang et al., 2018), the effect of which is to reduce the chance of subsequent mortality.

No mortality was recorded in the non-infected NN group throughout the trial, whereas in the other treatments mortality was first observed in the second week and was higher $(P<0.05)$ in SN compared with the SE and SP groups during the experiment. Severe mortality was observed in the third week of the experiment and $46.67 \%$ mortality was recorded in SN. Previously, Carina et al. (2000) noted $50 \%$ mortality in birds infected experimentally with Salmonella. Taghavi et al. (2008) noted that Salmonella infections at a young age can cause high mortality, whereas in older birds no clinical signs are seen. De Oliveira et al. (2014) found that mortality was not high in chicks that were challenged at 4 days of age, but performance was still compromised. Variation in mortality could be due to the ages and types of chickens, challenged organisms, and the dose and rearing status. The challenged birds had typical symptoms of salmonellosis, including prostration and ruffled feathers before death. Other clinical signs were increased thirst, reluctance 
to move, vent pasting and yellowish or greenish diarrhoea. Comparatively fewer severe clinical signs were observed in the SE and SP chickens. Mortality was not found in the non-infected group, and growth was retarded with decreased weekly weight gain in the chicks that survived infection. In contrast, low $(P<0.05)$ mortality rates and improved weight gains were noted in the SE and SP groups. These positive effects might be because of the effects of the supplemented probiotic and antibiotic products on the colonization of Salmonella in the gut.

\section{Conclusions}

Supplementation of $B$. subtilis facilitates growth in chickens through improvement in gut mucosal micro-architecture and improves the systemic immune response in birds. Moreover, B. subtilis produced results that were comparable with the antibiotic group and sustained better health status in broilers when they were challenged with Salmonella. Thus, the addition of $B$. subtilis to poultry feed is strongly recommended to avoid the side effects of antibiotics and to combat infectious agents better. Further studies are required to find the molecular pathways of $B$. subtilis QST-713 strain on activation of host immunity against pathogenic microorganisms.

\section{Acknowledgements}

This work was supported by HEC-Pakistan and the authors acknowledge Prof. Dr. Ashiq H. Cheema for providing professional writing services.

\section{Authors Contributions}

AS, AN, MD, and HFR established the experimental shed, arranged materials, and performed the methodology. $\mathrm{HZ}$ and AS analysed the data. AS, HMA, NM, TR and AR contributed to tissue collection, immunology, microscopy and the manuscript writeup.

\section{Conflict of Interest Declaration}

The authors declare that there is no conflict of interest.

\section{References}

Abo Ghanima, M.M., Abd El-Hack, M.E., Othman, S.I., Taha, A.E., Allam, A.A., Abdel-Moneim, A.M.E., 2020. Impact of different rearing systems on growth, carcass traits, oxidative stress biomarkers, and humoral immunity of broilers exposed to heat stress. Poult. Sci. 99(6), 3070-3078. DOI: 10.1016/j.psj.2020.03.011

Abudabos, A.M., Ali, M.H., Nassan, M.A. \& Saleh, A.A., 2019. Ameliorative effect of Bacillus subtilis on growth performance and intestinal architecture in broiler infected with Salmonella. Animals 9(4), 190. DOI: 10.3390/ani9040190

Arbab, S., Hafsa, Z., Muhammad, Y., Saima, M., Asim, A., Saima, A., Muhammad, A. \& Habib, R., 2017. Protective effect of sodium butyrate on growth performance, immune responses and gut mucosal morphometry in Salmonella-challenged broiler chickens. Int. J. Agri. Biol. 19, 1387-93. DOI: 10.17957/IJAB/15.0424

Audisio, M.C., Oliver, G. \& Apella, M.C., 2000. Protective effect of 10.1128/AEM.02747-16J96, a potential probiotic strain, on chicken infected with Salmonella pullorum. J. Food Prot. 63, 1333-37. DOI: 10.4315/0362-028X63.10.1333

Awad, W. A., Mann, E., Dzieciol, M., Hess, C., Schmitz-Esser, S., Wagner, M., \& Hess, M., 2016. Age-related differences in the luminal and mucosa-associated gut microbiome of broiler chickens and shifts associated with Campylobacter jejuni infection. Front. Cell. Infect. Microbiol. 6, 154. DOI:10.3389/fcimb.2016.00154

de Oliveira, J.E., Van der Hoeven-Hangoor, E., Van de Linde, I.B., Montijn, R.C. \& Van der Vossen, J.M.B.M., 2014. In ovo inoculation of chicken embryos with probiotic bacteria and its effect on posthatch Salmonella susceptibility. Poult. Sci. 93, 818-29. DOI: 10.3382/ps.2013-03409

Dersjant-Li, Y., Awati, A., Kromm, C. \& Evans, C., 2014. A direct fed microbial containing a combination of three-strain Bacillus spp. can be used as an alternative to feed antibiotic growth promoters in broiler production. J. Appl. Anim. Nutr. 2, 1-6. DOI: 10.1017/jan.2014.4

Dersjant-Li, Y., Gibbs, K., Awati, A. \& Klasing, K.C., 2016. The effects of enzymes and direct fed microbial combination on performance and immune response of broilers under a coccidia challenge. J. Appl. Anim. Nutr. 4,1-14. DOI: 10.1017/jan.2016.2

Lee, K.W., Kim, D.K., Lillehoj, H.S., Jang, S.I. \& Lee, S.H., 2015. Immune modulation by Bacillus subtilis-based direct-fed microbials in commercial broiler chickens. Anim. Feed. Sci. Technol. 200, 76-85. DOI: 10.1016/j.anifeedsci.2014.12.006

Nair, D.V.T \& Anup, K.J., 2019. Salmonella in poultry meat production. Food safety in poultry meat production. Food Microbiology and Food Safety series. Springer, Cham, Switzerland. Pp. 1-24. DOI: 10.1007/978-3-030-05011-5

Park, J.H. \& Kim, I.H., 2014. The effects of the supplementation of Bacillus subtilis RX7 and B2A strains on the performance, blood profiles, intestinal Salmonella concentration, noxious gas emission, organ weight and breast meat quality of broiler challenged with Salmonella typhimurium. J. Anim. Physiol. Anim. Nutr. 99, 326-34. DOI: $10.1111 /$ jpn.12248

Randall, L.P., Cooles, S.W., Coldham, N.C., Stapleton, K.S., Piddock, L.J. \& Woodward, M.J. 2006. Modification of enrofloxacin treatment regimens for poultry experimentally infected with Salmonella enterica serovar typhimurium 
DT104 to minimize selection of resistance. Antimicrobial Agents and Chemotherapy (AAC) 4030-37. DOI: 10.1128/AAC.00525-06

Saleem, G., Ramzaan, R., Khattak, F. \& Akhtar, R., 2016. Effects of acetic acid supplementation in broiler chickens orally challenged with Salmonella pullorum. Turk. J. Vet. Anim. Sci. 40, 434-43. DOI: 10.3906/vet1505-66

Shabani, R., Fakhraei, J., Yarahmadi, H.M. \& Seidavi, A., 2019. Effect of different sources of selenium on performance and characteristics of immune system of broiler chickens. Revista Brasileira de Zootecnia 48. https://www.scielo.br/pdf/rbz/v48/1806-9290-rbz-48-e20180256.pdf

Sikandar, A., Cheema, A.H., Younus, M., Aslam, A., Zaman, M.A. \& Rehman, T., 2012. Histopathological and serological studies on paratuberculosis in cattle and buffaloes. Pak. Vet. J. 32, 547-51.

Sikandar, A., Cheema, A.H., Adil, M., Younus, M., Zaneb, H., Zaman, A., Tipu, M.Y. \& Masood, S., 2013. Ovine paratuberculosis - A histopathological study from Pakistan. The J. Anim. Plant Sci. 23(3), 749-753.

Sikandar, A., Zaneb, H., Younus, M., Masood, S., Aslam, A., Shah, M. \& Rehman, H. 2017. Growth performance, immune status and organ morphometry in broilers fed Bacillus subtilis-supplemented diet. S. Afr. J. Anim. Sci. 47(3), 378-88.

Stefanello, C., Rosa, D.P., Dalmoro, Y.K., Segatto, A.L., Vieira, M. S., Moraes, M. L. \& Santin, E. 2020. Protected blend of organic acids and essential oils improves growth performance, nutrient digestibility, and intestinal health of broiler chickens undergoing an intestinal challenge. Frontiers in Veterinary Science 6, 491. DOI: 10.3389/fvets.2019.00491

Taghavi, A., Allan, B., Mutwiri, G., van Kessel, A., Willson, P., Babiuk, L., Potter, A. \& Gomis, S., 2008. Protection of neonatal broiler chicks against Salmonella Typhimurium septicemia by DNA containing CpG motifs. Avian Dis., 52, 398-406. DOI: 10.1637/8196-121907-Reg

Tarradas, J., Tous, N. \& Esteve-Garcia, E., 2020. The control of intestinal inflammation: A major objective in the research of probiotic strains as alternatives to antibiotic growth promoters in poultry. Microorganisms 8(2), 148. DOI: 10.3390/microorganisms8020148

Xie, S., Yang, F., Tao, Y., Chen, D., Qu, W., Huang, L., Liu, Z., Pan, Y. \& Yuan, Z., 2017. Enhanced intracellular delivery and antibacterial efficacy of enrofloxacin-loaded docosanoic acid solid lipid nanoparticles against intracellular Salmonella. Sci. Rep. 7, 41104. DOI: 10.1038/srep41104

Yang, J., Qian, K., Wang, C. \& Wu, Y., 2018. Roles of probiotic lactobacilli inclusion in helping piglets establish healthy intestinal inter-environment for pathogen defense. Probiotics Antimicrob. Proteins 10(2), 243-50. DOI: 1007/s12602-017-9273-y

Yang, Z., \& Liao, S. F., 2019. Physiological effects of dietary amino acids on gut health and functions of swine. Frontiers Veterinary Sci. 6. DOI: 10.3389/fvets.2019.00169

Yasmin, S., Nawaz, M., Anjum, A.A., Ashraf, K., Basra, M.A.R., Mehmood, A., Khan, I. \& Malik, F., 2019. Phytochemical analysis and In Vitro activity of essential oils of selected plants against Salmonella enteritidis and Salmonella gallinarum of poultry origin. Pak. Vet. J. 40(2), 139-144. DOI: 10.29261/pakvetj/2019.110

Zhang, W., Zhu, Y H., Zhou, D., Wu, Q., Song, D., Dicksved, J. \& Wang, J F. 2017. Oral administration of a select mixture of Bacillus probiotics affects the gut microbiota and goblet cell function in newly weaned MUC4 resistant pigs following Escherichia coli challenge. Appl. Environ. Microbiol. 83 (3), 2747-16. DOI: 10.1128/AEM.02747-16 